\title{
Nonuniqueness of the Potentials of Spin-Density-Functional Theory
}

\author{
Klaus Capelle \\ Instituto de Química de São Carlos, Universidade de São Paulo, \\ Caixa Postal 780, São Carlos, 13560-970 SP, Brazil \\ G. Vignale \\ Department of Physics and Astronomy, University of Missouri-Columbia, \\ Columbia, Missouri 65211
}

(Received 1 June 2000)

\begin{abstract}
It is shown that, contrary to widely held beliefs, the potentials of spin-density-functional theory (SDFT) are not unique functionals of the spin densities. Explicit examples of distinct sets of potentials with the same ground-state densities are constructed. These findings imply that the zero-temperature exchangecorrelation energy is not always a differentiable functional of the spin density. As a consequence, various types of applications of SDFT must be critically reexamined.
\end{abstract}

DOI: 10.1103/PhysRevLett.86.5546

PACS numbers: 71.15.Mb, 31.15.Ew, 75.10.Lp

The Hohenberg-Kohn (HK) theorem [1], which guarantees that the ground-state single-particle density uniquely determines all observables of a many-body system, is one of the most remarkable theorems of quantum mechanics. It is also at the heart of density-functional theory (DFT), one of the most popular many-body methods [2,3]. In the case of the original formulation of DFT, in which the basic variable is the particle density $n(\mathbf{r})$, the HK theorem can be cast in the form of two logically independent one-to-one maps [2]. Quantum mechanics guarantees that, for a given interaction and particle number, the potential in which the particles move determines the ground-state many-body wave function (via solution of Schrödinger's equation), which in turn determines the ground-state density (by simple integration). The essence of the original HK theorem is that both of these maps are invertible: the ground-state density $n(\mathbf{r})$ uniquely determines the groundstate wave function $\Psi\left(\mathbf{r}_{1}, \ldots, \mathbf{r}_{N}\right)$, which in turn determines, up to an additive constant, the potential [1-4],

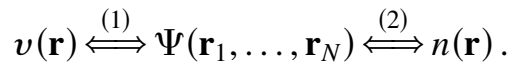

These abstract maps have found extremely important practical applications in the form of the Kohn-Sham (KS) formulation of DFT [5], which is used for almost all band-structure calculations in solid-state physics, and a rapidly increasing number of electronic-structure calculations in quantum chemistry. Many of these applications, however, do not employ the original formulation of DFT, but spin-density-functional theory (SDFT) [6], in which the fundamental variables are the spin-resolved particle densities $n_{\sigma}(\mathbf{r})$. In SDFT the map from spin densities to wave functions is easily established, but that from wave functions to potentials could not, in spite of considerable effort $[2,6,7]$ be proven and remains an, albeit popular, conjecture. In the early days of SDFT, von Barth and Hedin [6] already pointed out that the uniqueness of the spin-dependent potentials is not guaranteed, and ex- plicitly constructed two potentials which, when used in a one-body Hamiltonian, have common eigenstates. The question of whether a similar construction is possible in the many-body case, however, remained open, and even in the one-body case it was objected that these common eigenstates were not necessarily common ground states [2,7].

In this Letter, we settle these questions by first deriving a general equation [Eq. (2), below] whose solutions are the nonunique pieces of the potentials. This equation has two types of solutions, "accidental" ones and solutions that are connected with a special type of constant of motion. We then give a number of explicit and very simple examples for both types of solutions, and interpret the physics behind nonuniqueness in terms of a certain form of incompressibility.

The immediate and most important implication of these findings is that the universal energy functional $F$ (i.e., the expectation value of the sum of kinetic and interaction energy in the ground state $\Psi$ ) and its noninteracting counterpart $T_{s}$ are, generally, nondifferentiable functionals of the spin density. For, if they were differentiable, then their functional derivatives, evaluated at the ground-state densities, would uniquely determine the external and the KS potentials, respectively [see Eq. (7) below]. It follows that the exchange-correlation (xc) energy $E_{\mathrm{xc}}=F-$ $T_{s}-E_{H}$, where $E_{H}$ is the Hartree energy, is also nondifferentiable (barring a fortuitous cancellation of singularities), and the xc potential $v_{\mathrm{xc}}$ may have discontinuities at some densities. The existence of discontinuities in $v_{\mathrm{xc}}$ as a function of total particle number has been known for years as the source of the so-called "band-gap problem" in semiconductors $[8,9]$. Here we prove that a similar problem occurs in SDFT.

To obtain the condition for nonuniqueness let $v^{\prime}=$ $v+\Delta v$ and $\mathbf{B}^{\prime}=\mathbf{B}+\Delta \mathbf{B}$ be electrostatic and magnetic fields that are supposed to give the same (manybody) ground state $\Psi$ as $v$ and $\mathbf{B}$, and subtract the two corresponding many-body Schrödinger equations. The 
result is

$$
\int d^{3} r[\hat{n}(\mathbf{r}) \Delta v(\mathbf{r})-\hat{\mathbf{m}}(\mathbf{r}) \Delta \mathbf{B}(\mathbf{r})] \Psi=\Delta E \Psi .
$$

A necessary condition for nonuniqueness is thus that, given $\Psi$, one can find a linear combination of the density operators ( $\hat{n}$ and $\hat{\mathbf{m}}$ in the case of SDFT) of which $\Psi$ is an eigenfunction. This is automatically the case if a linear combination exists that is a constant of motion, since constants of motion commute with the Hamiltonian and thus have the same eigenfunctions (assumed nondegenerate for convenience). If the Hamiltonian expressed in terms of $v$ and $\mathbf{B}$ has a gap between its ground state and its first excited state, one can always make sure that $\Psi$ remains ground state of the new Hamiltonian, containing $\boldsymbol{v}^{\prime}$ and $\mathbf{B}^{\prime}$, by making the coefficients of the linear combination, $\Delta v$ and $\Delta \mathbf{B}$, sufficiently small.

From any extensive constant of motion (i.e., one which is a linear combination of the density operators) one can thus systematically construct families of potentials with the same ground state. As a first example consider a system with an energy gap, for which the total magnetization $\hat{M}_{z}=\int d^{3} r \hat{m}_{z}(\mathbf{r})$ is a constant of motion. The choice $\Delta v=0, \Delta \mathbf{B}=\bar{B} \mathbf{u}_{z}=$ const, where $\mathbf{u}_{z}$ is a unit vector in the $z$ direction, then is clearly a suitable linear combination, as long as $\bar{B}$ is not large enough to induce level crossings. The presence of a gap is crucially important, since it guarantees that for sufficiently small values of $\bar{B}$ such level crossings do not take place, so that $\Psi$ remains the ground state.

Examples for nonuniqueness obtained from constants of motion are below referred to as systematic nonuniqueness [10]. Apart from these, Eq. (2) may, under suitable circumstances, also permit solutions not systematically associated with conserved quantities. To construct an example for such accidental nonuniqueness, first recall that in conventional SDFT the spin densities $n_{\sigma}(\mathbf{r})=$ $\sum_{n}^{N_{\sigma}} \phi_{n \sigma}^{*}(\mathbf{r}) \phi_{n \sigma}(\mathbf{r})$ are calculated from the solutions of the spin-dependent KS equation,

$$
\left[-\frac{\hbar^{2}}{2 m} \nabla^{2}+v_{s, \sigma}(\mathbf{r})\right] \phi_{n \sigma}(\mathbf{r})=\epsilon_{n \sigma} \phi_{n \sigma}(\mathbf{r}),
$$

where $N_{\sigma}$, the number of particles with spin $\sigma$, is determined by filling the $N$ lowest eigenfunctions $\phi_{n \sigma}$ in sequential order. Here we restricted ourselves to collinear spin configurations, so that the magnetic field can be written as $B_{s, z}=\left[v_{s, \downarrow}-v_{s, \uparrow}\right] / 2 \mu$, where $\mu=q \hbar /(2 m c)$ is the Bohr magneton. If the number of filled spin up levels is equal to that of filled spin down levels, the system is unpolarized $\left(N_{\uparrow}=N_{\downarrow}\right)$, while it acquires a finite spin polarization if they are different. The maximum possible spin polarization is realized if all particles have the same spin (say spin up), so that $n_{\uparrow}=n$ and $n_{\downarrow}=0$.

In the case of a fully polarized system (e.g., saturated ferromagnetism), the $N$ lowest eigenvalues of the spin up $\mathrm{KS}$ equation are all lower than the lowest eigenvalue of the spin down KS equation, so that no eigenfunction of the latter is occupied. The full density is thus obtained from the self-consistent solution of

$$
\left[-\frac{\hbar^{2}}{2 m} \nabla^{2}+v_{s, \uparrow}(\mathbf{r})\right] \phi_{n \uparrow}(\mathbf{r})=\epsilon_{n \uparrow} \phi_{n \uparrow}(\mathbf{r}),
$$

with $n(\mathbf{r})=n_{\uparrow}(\mathbf{r})=\sum_{n}^{N} \phi_{n \uparrow}^{*}(\mathbf{r}) \phi_{n \uparrow}(\mathbf{r})$. In this case the spin up KS potential of SDFT already determines the full particle density $n(\mathbf{r})$. The spin down potential, on the other hand, is a completely arbitrary function of $\mathbf{r}$, as long as its lowest eigenvalue $\epsilon_{0 \downarrow}$ is higher than the $N$ th eigenvalue of the corresponding spin up equation, $\epsilon_{N \uparrow}$ (which is precisely the condition for the system to remain fully polarized) [11]. The set of spin densities $\left\{n_{\uparrow}=n, n_{\downarrow}=0\right\}$ thus does not uniquely determine the set of potentials $\left\{\boldsymbol{v}_{s \uparrow}, \boldsymbol{v}_{s \downarrow}\right\}$, because there trivially is an infinite number of possible spin down potentials. Since spin densities and wave functions are still in one-to-one correspondence, this implies that the potentials of SDFT are not determined completely by either the KS Slater determinant $\Phi$ or the full many-body ground state $\Psi$.

In the less extreme case of a partially polarized system this simple example for accidental nonuniqueness breaks down, but it is not hard to show that partial polarization does not restore uniqueness. Consider thus, as our next example, a given representation of the ground state KS Slater determinant in terms of single-particle orbitals $\phi_{n}$. In ordinary DFT, based on the density only, each of these orbitals determines the corresponding KS potential (up to the irrelevant additive constant $\epsilon_{n}$ which determines the zero of energy) according to

$$
v_{s}(\mathbf{r})=\epsilon_{n}-\frac{\hat{t} \phi_{n}(\mathbf{r})}{\phi_{n}(\mathbf{r})},
$$

where $\hat{t}$ is the single-particle kinetic energy operator. Equation (5) is simply the KS equation solved for $v_{s}(\mathbf{r})$ [12]. If one now tries to repeat this reasoning in the spin-dependent case, one must solve the KS equation of SDFT for the external potentials $v_{s, \uparrow}(\mathbf{r})$ and $v_{s, \downarrow}(\mathbf{r})$ in terms of the single-particle orbitals $\phi_{n \dagger}(\mathbf{r})$ and $\phi_{n \downarrow}(\mathbf{r})$,

$$
v_{s, \sigma}(\mathbf{r})=\epsilon_{n \sigma}-\frac{\hat{t} \phi_{n \sigma}(\mathbf{r})}{\phi_{n \sigma}(\mathbf{r})} .
$$

Again, any of the single-particle orbitals determines the corresponding potential up to a constant, $\epsilon_{n \sigma}$, which remains unspecified. But, unlike in the previous case, one can choose only one constant freely by adjusting the zero of energy. That is, given $\phi_{n \uparrow}(\mathbf{r})$, say, one can completely determine $v_{s, \uparrow}(\mathbf{r})$ by fixing the zero of energy, but then $\phi_{n \downarrow}(\mathbf{r})$ determines $v_{s, \downarrow}(\mathbf{r})$ only up to an unknown constant. Although this constant is physically relevant since it rigidly shifts spin up levels with respect to spin down levels, it is manifestly not knowable in terms of only the orbitals. Consequently, the KS Slater determinant does not completely determine the spin-dependent potentials. By appealing to the second part of the HK theorem one readily concludes that the same holds for the many-body wave function $\Psi$. The only caveat to the above construction is that for the 
original KS determinant to remain the ground state after adding the free constant to $v_{s, l}$, this shift must not induce the presence of new (previously unoccupied) singleparticle orbitals in the Slater determinant. As above, this is guaranteed if the KS N-particle ground state is separated from the first excited state by a gap, and the shift of $v_{s, \downarrow}$ is sufficiently small compared to this gap.

Adding a constant term, $\bar{v}$, to $v_{s, \downarrow}(\mathbf{r})$ amounts to adding $\bar{v} / 2$ to the electrostatic potential $v_{s}(\mathbf{r})$ and $\bar{v} / 2 \mu$ to the magnetic field $B_{s, z}(\mathbf{r})$. The nonuniqueness associated with the fact that there is only one zero of energy, but two constants to be determined in Eq. (6), has thus brought us back to a particular example of systematic nonuniqueness, in which the linear combination in Eq. (2) is given by $\Delta v=\bar{v} / 2$ and $\Delta \mathbf{B}=[\bar{v} /(2 \mu)] \mathbf{u}_{z}$. In general, we expect that systematic nonuniqueness is the only generic source of nonuniqueness, while accidental nonuniqueness requires certain "pathological" features, such as complete spin polarization.

Reflecting on the previous examples, what all cases of nonuniqueness, accidental or systematic, have in common is that the wave functions do not change if certain changes are made to the potentials. This implies a degree of "rigidity" or "incompressibility" of the former with respect to the latter. The relation to incompressibility is, in fact, more than a mere analogy: an essential feature of all nontrivial examples of nonuniqueness (i.e., all except the one mentioned in footnote [10]) is a gap between ground and first excited state, as a consequence of which sufficiently small changes to the potentials cannot bring down an excited state to have lower energy than the ground state. The existence of a gap between ground and first excited state, on the other hand, is tantamount to incompressibility in the usual (quantum liquid) sense of the word.

Another way to look at the above findings is to recall that the KS potentials can be expressed as

$$
v_{s, \sigma}(\mathbf{r})-\mu=-\frac{\delta T_{s}\left[n_{\uparrow}, n_{\downarrow}\right]}{\delta n_{\sigma}(\mathbf{r})},
$$

where $\mu$ is the chemical potential and $T_{s}$ the noninteracting kinetic energy functional of Refs. [5] and [6]. Nonuniqueness of $v_{s, \sigma}$ implies that $T_{s}$ does not possess a functional derivative for all spin densities. This result could have been anticipated from the Oliver-Perdew [13] spin-scaling relation $T_{s}\left[n_{\uparrow}, n_{\downarrow}\right]=\left(T_{s}\left[2 n_{\uparrow}\right]+T_{s}\left[2 n_{\downarrow}\right]\right) / 2$, which connects the $T_{s}$ functional of SDFT, $T_{s}\left[n_{\uparrow}, n_{\downarrow}\right]$, to that of ordinary DFT, $T_{s}[n]$. Changing the spin density at constant particle density means that at least one spin must be flipped: this changes the total particle numbers $N_{\uparrow}$ and $N_{\downarrow}$, causing the DFT functionals on the right-hand side of the relation to be affected by their well-known derivative discontinuities. An obvious extension of Eq. (7), with $v_{s}$ and $T_{s}\left[n_{\uparrow}, n_{\downarrow}\right]$ replaced by the external potential and $F\left[n_{\uparrow}, n_{\downarrow}\right]$, respectively, proves that nonuniqueness implies nondifferentiability of $F$, too. From this we conclude that the xc potential $v_{\mathrm{xc}, \sigma}(\mathbf{r})=\delta\left\{F-T_{s}-E_{H}\right\} / \delta n_{\sigma}(\mathbf{r})$ is generally a discontinuous functional of the spin density.
The above conclusions have been reached within the frame of the zero-temperature (ground-state) formalism, with integral occupations of the KS orbitals. It is natural, at this point, to ask whether all these difficulties could be avoided by going to finite temperature or fractional orbital occupation [8] ensembles. As in the analogous band-gap problem we believe that there is no easy way to fix the problem. For example, it is true that in the finite temperature formalism the thermodynamic xc potential becomes a differentiable function of particle number, due to the finite fractional occupation of states above a band gap [14]. But this means only that the $T=0$ discontinuity is spread over a finite (infinitesimal for $T \rightarrow 0$ ) range of fractional particle numbers. As long as $k_{B} T$ is much smaller than the gap, the quantity of physical interest remains the total change of $v_{\mathrm{xc}}$ across that range, which is essentially the same as the $T=0$ discontinuity, and is, unfortunately, unknown.

In the remainder of this paper we briefly describe several situations which are directly affected by the nonuniqueness of the potentials: (i) calculation of excited states within SDFT, (ii) determination of exact KS potentials from numerically exact densities, (iii) perturbative strategies to construct approximate density functionals, (iv) calculations of one-electron spin gaps in half-metallic ferromagnets, and (v) the optimized-effective potential method of SDFT.

(i) It is sometimes stated [2,3] that the ground-state density of DFT in principle contains all information about excited states, too, since it determines the external potential, which in turn determines the entire energy spectrum. From the above it is clear that this statement does not hold in SDFT in the presence of a magnetic field, since the spin densities do not fully determine the external potentials $v(\mathbf{r})$ and $\mathbf{B}(\mathbf{r})$.

(ii) Recently several methods for constructing accurate KS potentials, using as input highly precise numerical densities (obtained from Monte Carlo or configuration interaction calculations), have been proposed [15]. Uniqueness of the resulting KS potentials is taken for granted in these prescriptions. Clearly, this holds only in the original formulation of DFT, but not in the (much more widely used) SDFT. The generalization of these methods to determine the KS potentials of SDFT must thus be reconsidered.

(iii) A useful method for obtaining LDA-type approximations for the $\mathrm{xc}$ functional is based on perturbative diagrammatic calculations of the xc energy of the electron gas $[2,16]$. The results of such calculations are necessarily obtained in terms of the potentials acting on the unperturbed system. As long as the first map of the HK theorem holds, they are thus automatically also functionals of the densities. Obviously this ceases to be true if that map is no longer available. It follows from the above that the SDFT counterpart of this procedure works only because the electron gas does not have a gap between its ground and first excited state. Attempts to apply this procedure to systems with a gap (such as the superconducting electron 
gas considered in Ref. [16]) thus face unanticipated and fundamental problems.

(iv) Half-metallic ferromagnets have only electrons of one spin orientation at the Fermi level, while electrons of the opposite spin orientation are in a full valence band below the Fermi level. An important quantity is the "spin gap," i.e., the energy required to flip a minority spin electron from the top of the occupied valence band to the Fermi level of majority spins. In principle, this quantity could be calculated from SDFT because it is the difference between the energies of two ground states with different values of $S_{z}$. However, in view of our findings (see, in particular, our third example above), it is very likely that any SDFT calculation of the spin gap will miss a contribution due to a discontinuity in the exchange-correlation potential.

(v) In the optimized-effective potential method one minimizes an energy functional that is an explicit functional of the single-particle orbitals and only an implicit functional of the densities. In order to calculate the functional derivative with respect to the densities, one then employs the functional chain rule to write $[17,18]$

$$
\begin{aligned}
v_{\mathrm{xc}}[n](\mathbf{r})= & \frac{\delta E_{\mathrm{xc}}\left[\left\{\phi_{n}\right\}\right]}{\delta n(\mathbf{r})} \\
= & \int d r^{\prime} \int d r^{\prime \prime} \sum_{n} \frac{\delta E_{\mathrm{xc}}\left[\left\{\phi_{n}\right\}\right]}{\delta \phi_{n}\left(\mathbf{r}^{\prime}\right)} \\
& \times \frac{\delta \phi_{n}\left(\mathbf{r}^{\prime}\right)}{\delta v_{s}\left(\mathbf{r}^{\prime \prime}\right)} \frac{\delta v_{s}\left(\mathbf{r}^{\prime \prime}\right)}{\delta n(\mathbf{r})}+\text { c.c. }
\end{aligned}
$$

The first functional derivative on the right-hand side can be calculated explicitly, the second is easily found from perturbation theory, and the last is usually identified with an inverse response function. However, if the KS potentials are not unique, this inverse does not exist for all densities. In practice one does not explicitly calculate the derivative $\delta v_{s}\left(\mathbf{r}^{\prime \prime}\right) / \delta n(\mathbf{r})$, but expresses it in terms of the noninteracting response function. For this procedure to work in SDFT and to uniquely determine $v_{\mathrm{xc}, \sigma}(\mathbf{r})$, one must supplement it by additional information on $v_{s, \sigma}(\mathbf{r})$, to eliminate its nonuniqueness. The relative additive constant discussed below Eq. (6), for example, can be fixed by considering the proper decay of the KS potentials as $r \rightarrow \infty$, for a finite system.

Our conclusions (i) to (v) above were formulated explicitly for SDFT, but nonuniqueness is not limited to that formulation of DFT. In fact, similar examples for nonuniqueness have recently also been found by one of us in current-density functional theory [19].

In summary, we have shown, both by explicit examples and by general considerations, that, unlike in ordinary DFT, the effective and external potentials of SDFT are not uniquely determined by the spin densities alone. Nonuniqueness can arise accidentally, via special features of the ground state, and systematically, via extensive constants of motion. As a consequence many previous applications of SDFT must be critically reexamined.
Similar conclusions have recently and independently been reached by Eschrig and Pickett [20].

K. C. thanks L. N. Oliveira, M. Lüders, and E. K. U. Gross for valuable discussions, the Physics Department at the University of Columbia, Missouri, for generous hospitality, and the FAPESP for financial support. G. V. acknowledges support from NSF Grants No. DMR 9706788 and No. DMR-0074959.

[1] P. Hohenberg and W. Kohn, Phys. Rev. 136, B864 (1964).

[2] R. M. Dreizler and E. K. U. Gross, Density Functional Theory (Springer-Verlag, Berlin, 1990).

[3] R. G. Parr and W. Yang, Density-Functional Theory of Atoms and Molecules (Oxford University Press, Oxford, 1989).

[4] M. Levy, Adv. Quantum Chem. 21, 69 (1990).

[5] W. Kohn and L. J. Sham, Phys. Rev. 140, A1133 (1965).

[6] U. von Barth and L. Hedin, J. Phys. C 5, 1629 (1972).

[7] A. K. Rajagopal, Adv. Chem. Phys. 41, 59 (1980).

[8] J. P. Perdew, R. G. Parr, M. Levy, and J. L. Balduz, Phys. Rev. Lett. 49, 1691 (1982).

[9] J. P. Perdew and M. Levy, Phys. Rev. Lett. 51, 1884 (1983).

[10] The familiar trivial nonuniqueness of the potential $v(\mathbf{r})$ with respect to addition of an arbitrary constant is another example, associated with the constant of motion $\hat{N}$, the total particle number.

[11] The existence of a fully polarized ground state implies the presence of an energy gap between this state and its first not fully polarized excited state a collinear magnetic field can produce, characterized by a single flipped spin. (Noncollinear fields could produce gapless magnons, but are explicitly excluded from the present discussion in terms of $v_{s, \uparrow}$ and $v_{s, \downarrow}$ only.)

[12] Equation (5) can break down if $\phi_{n}$ vanishes on a set of measures different from zero. In this case one is dealing not with one quantum system, but with two totally disconnected systems, a situation we do not study in the present paper.

[13] G. L. Oliver and J. P. Perdew, Phys. Rev. A 20, 397 (1979).

[14] T. K. Ng, Phys. Rev. B 39, 1369 (1989).

[15] Y. Wang and R. G. Parr, Phys. Rev. A 47, 1591 (1993); Q. Zhao, R. C. Morisson, and R. G. Parr, Phys. Rev. A 50, 2138 (1994); A. Görling, Phys. Rev. A 46, 3753 (1992); F. Aryatsetiawan and M. J. Stott, Phys. Rev. B 38, 2974 (1988); R. van Leeuwen and E. J. Baerends, Phys. Rev. A 49, 2421 (1994); A. Holas and N. H. March, Phys. Rev. A 44, 5521 (1991); A. M. Lee and N. C. Handy, Phys. Rev. A 59, 209 (1999).

[16] S. Kurth, M. Marques, M. Lüders, and E. K. U. Gross, Phys. Rev. Lett. 83, 2628 (1999).

[17] T. Grabo, T. Kreibich, S. Kurth, and E. K. U. Gross, in Strong Coulomb Correlations in Electronic Structure: Beyond the Local Density Approximation, edited by V.I. Anisimov (Gordon \& Breach, Tokyo, 1998).

[18] A. Görling and M. Levy, Phys. Rev. A 50, 196 (1994).

[19] G. Vignale (unpublished).

[20] H. Eschrig and W.E. Pickett, Solid State Commun. 118, 123 (2001). 